\title{
Da Montecatini ad Altopascio: Firenze senza Dante
}

\begin{abstract}
Gli ultimi cinque anni della vita di Dante possono essere visti, dal punto di vista del coinvolgimento politico di Firenze, come una fase intermedia tra due grandi battaglie che si risolsero nella sconfitta della parte guelfa in Italia centrale. I capi ideologici e militari della parte guelfa, il papa e il re di Sicilia, cercarono di sfruttare i comuni guelfi per i loro scopi. I comuni, da parte loro, cercarono di venire incontro alle richieste di questi ultimi ma anche alle loro esigenze locali e regionali. L'articolo propone un bilancio delle diverse necessità di Firenze, principale città guelfa e punto di riferimento per Dante, nonostante la sua condizione di exul immeritus.
\end{abstract}

The last five years in Dante's life may be seen, from the vantage point of Florence's political involvement, as the interim between two major battles that ended in defeat of the Guelf party in middle Italy. The ideological and the military heads of this party, the pope and the king of Sicily, tried to use the Guelf communes for their own means. The communes on the other hand tried to cope with the "masters" requirements as well as with their own local and regional needs. This article tries to assess the diverging needs of Florence as the most prominent Guelf city - and mainly as the point of reference for Dante even though his state of exul immeritus.

Parole chiave: Firenze, Papa Giovanni XXII, storia politica, Roberto d'Angiò, parte guelfa.

Mi è stato affidato il compito di abbozzare il contesto politico di Firenze nell'ultimo lustro della vita di Dante. ${ }^{1}$ Questo contesto sarà presentato alla luce delle

1 Questo mio contributo è nato dalla mia tesi di laurea, presentata all'Università di Colonia nel 2011 e diretta dal professor Ludwig Vones.

Annotazione: Tengo molto a ringraziare Giuliano Milani, Antonio Montefusco e Gaia Tomazzoli per la rilettura e i suggerimenti.

Andreas Kistner, Heinrich Heine Universität Düsseldorf

○ Open Access. ( 2020 Andreas Kistner, published by De Gruyter. (c) BY This work is licensed under the Creative Commons Attribution 4.0 International License (CC BY 4.0). https://doi.org/10.1515/9783110590661-026 
grandi linee della politica guelfa; ${ }^{2}$ l'ultimo lustro della vita del poeta coincide infatti con i primi cinque anni del pontificato del primo "vero" papa avignonese: Giovanni XXII. Il papa tuttavia costituisce solo uno dei poli che compongono il triangolo guelfo, vale a dire quello ideologico-teologico: gli altri sono Firenze - polo finanziario - e Napoli - centro costante della forza militare sulla penisola italiana. Limitarsi solo ai primi due poli significa rischiare di non considerare una parte importante della politica italiana, ossia la potenza militare. Tanto più che dal 1313 al 1322 Firenze si diede in signoria a Roberto, e che Roberto dimorò ad Avignone presso il papa dal 1319 fino al 1324.

Nelle pagine che seguono procederemo nel modo seguente: dopo alcune considerazioni sulle fonti disponibili, accenneremo alla situazione generale della politica italiana attorno al momento dell'elezione di papa Giovanni XXII papa severamente criticato da Dante, come si dirà; è un momento in cui è attiva una forte cooperazione tra il braccio militare del papa, re Roberto, e Firenze. Per seguire il periodo successivo cambieremo punto di vista e ci concentreremo sull'azione militare di Firenze nei confronti di Genova, della Lombardia e della Toscana. Nell'ultimo paragrafo si tenterà di delineare alcune linee generali della relazione tra il papa e Firenze, la patria del poeta.

\section{Osservazioni sulle fonti}

Quando si fanno ricerche sul papato del secolo XIV non si può fare a meno di consultare i registri di lettere pontificie da tempo oggetto di pubblicazioni da parte dell'École française de Rome. ${ }^{3}$ Seguendo questa serie per il periodo che

\footnotetext{
2 Per quanto riguarda i termini e il loro valore significativo si rinvia a Herde, Guelfen und Neoguelfen; Herde, Guelfen und Gibellinen, e alle voci corrispondenti di Franco Cardini nel Lexikon des Mittelalter IV (1989), coll. 1436-1438 e 1763-1765. Recentemente è stato importante l'intervento di Raveggi, L'Italia dei guelfi; cfr. anche Maire Vigueur, Nello Stato, p. 769: «come ben sappiamo, il guelfismo è una nozione fluttuante e la qualifica di guelfo si applica, secondo i momenti e le circostanze, a una gamma abbastanza ampia di scelte compiute nel quadro della politica sia interna che esterna delle città e degli altri soggetti politici. (...) Nel quadro dell'Italia centrale, il guelfismo caratterizza senz'altro la politica estera delle città fedeli, nella lunga durata, ad un sistema di alleanza dominato della Chiesa, da Firenze e/o dalla monarchia angioina, e possiamo anche etichettare come guelfo il personale politico che gode della fiducia dell'una o dell'altra di queste tre entità - meglio ancora se riceve incarichi ufficiali da parte di esse».

3 Nel nostro caso si tratta di Jean XXII, Lettres communes e Jean XXII, Lettres secrètes et curiales relatives á la France - entrambi i corpora consultati grazie al database Ut per litteras apostolicas ospitato su <apps.brepolis.net/litpa/Pontificates.aspx>.
} 
va dal primo gennaio 1316 fino al 31 dicembre 1321, pur limitandosi alle sole lettere emesse da Giovanni XXII, ci si trova a considerare ben 16272 lettere, delle quali 237 menzionano Firenze (Florenti*). Si tratta di lettere "fiorentine" in senso molto ampio: provvisioni di benefici per chierici fiorentini che risiedono altrove, provvisioni di benefici per chierici che risiedono a Firenze, sostegni papali alle società bancarie di Firenze (i Bardi soprattutto) e pagamenti in moneta fiorentina. E tutto questo corrisponde solo all'1,5\% delle lettere papali di questo periodo che sono state pubblicate. Più precisamente per il periodo 1322-1326 le lettere che si riferiscono a Firenze sono 234 su un totale di 14075 lettere (1,7\%); per il periodo 1327-1331 sono 409 su 21255 (1,9\%); negli anni 1332-1334 sono $100 \mathrm{su} 8954$ (1,1\%). Le lettere “secrete” relative alla Francia del periodo 13161321 sono 8; negli anni 1322-1326 sono 11; tra il 1327 e il 1331 arrivano a 18, e dal 1332 al 1334 tornano a essere 6. L'ultimo lustro della vita di Dante, per quanto riguarda le relazioni fiorentino-papali, è segnato dunque da una certa freddezza. ${ }^{4}$

L’analisi condotta si limita alle lettere edite sinora, cioè alle lettere politiche relative alla solo Francia. Quelle relative ad altri paesi non sono state oggetto di iniziativa editoriale fino ad oggi; è possibile dunque che i risultati cambino una volta che tali lettere saranno pubblicate, anche se, a mio modo di vedere, probabilmente non molto. Purtroppo per la preparazione di questo mio contributo non ho potuto consultare i registri inediti conservati presso l'Archivio Segreto Vaticano. Le vecchie pubblicazioni di Preger e Reinkens permettono di rimediare in parte a questa lacuna, ma da esse non possono essere estratti dati quantitativi. ${ }^{5}$

Per quanto riguarda le fonti narrative è molto importante la Nuova Cronica di Giovanni Villani; ${ }^{6}$ le forzature del posizionamento politico del Villani ${ }^{7}$ posso-

4 Per uno sguardo complessivo sulle iniziative papali attraverso i registri secreti cfr. Zanke, Johannes XXII. Si vedano anche le recensioni di R. Lützelschwab (<http://www.sehepunkte.de/ 2014/12/24267.html >) e K. Hitzbleck (<http://www.perspectivia.net/publikationen/francia/ francia-recensio/2016-1/ma/zahnke_hitzbleck >).

5 Preger, Die Anfänge; Preger, Reinkens, Die Verträge; Riezler, Vatikanische Akten.

6 Si utilizza l'edizione di Giuseppe Porta del 1991 (Villani, Nuova Cronica). Il Porta propose un nuovo ordinamento dei capitoli e dei libri rispetto all'edizione "classica" di Dragomanni del 1845.

7 Come sottolinea Rodolico nella prefazione alla sua edizione della Cronaca Fiorentina di Marchionne di Coppo Stefani, p. LXXXVII. Cfr. anche Green, Chronicle, p. 16: «for Giovanni Villani, the Church remained the embodiment of the forces of the righteousness in history, in spite of the aberrations of its Pastors, and the Emperors of the Hohenstaufen line (...) the incarnation of evil in so far as they opposed its interests or defied its pronouncements». Non si possono verificare sempre tali divergenze tra le fonti. 
no essere comprese alla luce della sua biografia. ${ }^{8}$ Questi scrisse la sua Nuova Cronica a partire degli anni 1320-30 del secolo XIV, ${ }^{9}$ dunque in contemporanea o poco dopo gli eventi qui discussi. In alcuni casi si farà ricorso anche alla cronica di Marchionne di Coppo Stefani, fondata sul testo di Villani ma caratterizzata da alcune aggiunte; il suo orizzonte strettamente fiorentino limita però le nostre possibilità di usare questa fonte scritta nella prima metà degli anni $1380 .^{10}$

\section{La situazione attorno all'elezione di Jacques Duèse}

Dopo la morte di Clemente V (20 aprile 1314), profetizzata da Nicolò IV nell'Inferno, i conflitti interni alle città ricominciarono ${ }^{11}$ e la parte guelfa subì una serie di sconfitte: Lucca fu conquistata da Uguccione della Faggiuola il 14 giugno $1314^{12}$ e, un anno più tardi, il 29 agosto 1315, Firenze subì la sconfitta di Montecatini - evento luttuoso che si può forse intravedere dietro la funesta profezia che Forese Donati consegna a Dante nel XXIII canto del Purgatorio (vv. 106-108). Nonostante Pietro, detto “tempesta” (fratello di Roberto d'Angiò che dal 1313 era anche signore di Firenze), fosse morto durante questa battaglia, ${ }^{13}$ Roberto non diede seguito al desiderio di vendetta dei fiorentini, e per questa ragione cominciò a essere visto come un vigliacco e come un avaro che non intendeva pagare le truppe mercenarie. ${ }^{14}$ Queste accuse nei confronti di

8 Green, Chronicle, pp. 11-13.

9 Green, Chronicle, p. 9.

10 Marchionne di Coppo Stefani, Cronaca fiorentina, pp. LXXXVII-XCII; CIII; CVIII. Per una valutazione generale di questa fonte si vedano De Vincentiis, Scrittura storica; Green, Chronicle, p. 90 .

11 Heckmann, Stellvertreter, p. 428; Cognasso, L’unificazione, pp. 15-34; 50-57; 60; 65-66; 93; Simeoni, Le signorie, p. 88; Bock, Kaisertum, p. 44.

12 Per questo personaggio si veda la voce di C. E. Meek nel DBI (<http://www.treccani.it/ enciclopedia/uguccione-della-faggiuola_(Dizionario-Biografico >); cfr. anche Green, Castruccio Castracani, capitolo II.

13 Davidsohn, Geschichte, III, pp. 581-584. Per gli antecedenti della battaglia, cfr. ivi, p. 576. Il malcontento fiorentino fu acceso ulteriormente dalle esigenze finanziarie di Filippo da Taranto (cfr. Acta Arag. II, p. 553, n. 362). Dall'altro lato questa sconfitta ebbe come conseguenza che Firenze fu trascinata ancora di più in «the old pattern of guelf and ghibelline hostility» (Partner, Florence and Papacy, p. 83).

14 Davidsohn, Geschichte, III, p. 586. Questo malcontento fu forse soltanto un fenomeno fiorentino, come suggerisce Abulafia, The Western Mediterranean Kingdoms, p. 139: «Robert 
Roberto erano largamente diffuse, e confluirono anche nella Commedia, dove Carlo Martello lamenta la parca discendenza di una stirpe tanto larga e la mancata lungimiranza del fratello nei confronti dell'avarizia dei catalani (Par. VIII, 67-84).

Con la morte di Enrico VII nel 1313, del resto, era venuto a mancare il nemico comune, e perciò anche una comune direzione della politica. La mancata prosecuzione della guerra da parte di Roberto peggiorò ulteriormente i rapporti tra i due alleati. ${ }^{15}$

L'assunzione del potere a Lucca da parte di Castruccio Castracani ${ }^{16}$ e il rovesciamento del governo a Pisa non cambiarono la situazione generale e gran parte d'Italia si trovò a essere dominata da signori ghibellini, come i Visconti a Milano e i della Scala a Verona, che cercavano di allargare i loro domini. ${ }^{17}$ In questa situazione Jacques Duèse fu eletto al soglio pontificio il 7 agosto $1316 .{ }^{18}$

L'inizio del suo pontificato fu segnato da una speranza di miglioramento: negli anni 1316-1317 le guerre si arrestarono. Dopo un altro cambio di regime i pisani mandarono una legazione a Napoli per trattare la pace; il 12 agosto 1316 fu firmato un primo trattato, e il 12 maggio 1317 anche gli altri comuni dell'alleanza angioina accettarono - a malincuore ${ }^{19}$ - la pace. ${ }^{20}$ Se tuttavia Roberto

proved able just to hold his position, exercising influence as leader of the Guelf factions from Piedmont to Tuscany».

15 Kelly, The New Solomon, p. 228.

16 Green, Castruccio Castracani, pp. 30-60. La nomina a vicario generale dell'impero gli comportò, nel contesto regionale, una più forte legittimazione. Nel 1320 fu nominato da Federico il Bello e nel 1324 da Lodovico IV (cfr. MGH, Const. V, n. 570, p. 458; n. 573, p. 460; n. 925, p. 764). Per quanto riguarda la ricerca sul vicariato imperiale si veda infra, nota 27.

17 Jugie, Un Quercynois, p. 75; Mollat, Les papes d'Avignon, p. 186, e più recentemente: Favier, Les papes d'Avignon, pp. 43-48.

18 Mollat, Les papes d'Avignon, pp. 156-57. Tabacco, Egemonie sociali, p. 327: «la "sede romana”, operante ora da Avignone, con una tranquillità e una sicurezza quali in Roma non aveva mai conosciuto, riassunse energicamente il comando del fronte guelfo. Il combattivo pontefice (1316-34) inserì il problema italiano nel contesto europeo. La soluzione del problema italiano doveva essere guelfo-angioina, in un'Europa diretta dal papato e dalla casa di Francia: domus Franciae benedicta, la casa a cui appartenevano così i Capetingi regnanti a Parigi, come gli Angioini di Napoli, discendenti gli uni dal santo re Luigi IX, gli altri dal suo audace fratello Carlo d'Angiò».

19 Villani, Nuova cronica, X 82, p. 287: «e con tutto che per gli Guelfi malvolentieri si facesse per la sconfitta ricevuta da lloro».

20 Davidsohn, Geschichte, pp. 605-06; Tabacco, La casa di Francia, p. 153. Secondo le Cronache Senesi, p. 111, le prime trattative del trattato di pace si fecero senza conoscenza degli alleati: «e none aveva detto niente a nisuno, e masime a noi Sanesi, e' quai eravamo cholegati cho lui». 
aveva concluso questi trattati sulla base dei suoi interessi, ${ }^{21}$ i comuni guelfi, da parte loro, non erano pronti a mettere termine alla guerra.

In questo frangente il ruolo di Roberto fu di garantire la sicurezza con mezzi militari, mentre quello del papa fu quello di mettere a disposizione una rete diplomatica. I comuni guelfi, e Firenze tra gli altri, rimasero insoddisfatti, mentre Roberto $\mathrm{fu}$ in grado di approfittare della situazione: cessata la minaccia imperiale e conclusa la dispendiosa guerra in Toscana e Lombardia, si riapriva per lui la possibilità di avvantaggiarsi di un contributo pisano per il conseguimento di quello che per lui restava l'obiettivo principale: la riconquista della Sicilia. Firenze e gli altri comuni, dal canto loro, vedevano Roberto come un re interessato a utilizzare strumentalmente l'alleanza guelfa per difendere il suo regno dalla minaccia imperiale senza tener conto degli interessi dei suoi alleati. ${ }^{22}$ D’altro canto il papa stesso sollecitava già da qualche tempo il re a fare la pace; le difficoltà finanziarie furono un argomento importante. ${ }^{23}$ Ma Giovanni XXII percepiva anche il conflitto delle parti come un danno per tutta l'Italia e come ostacolo a un possibile ritorno della Curia Romana a Roma. ${ }^{24}$

Firenze godette di quattro anni di pace, ma si trovò comunque costretta a contribuire alle esigenze militari degli alleati, ${ }^{25}$ in primo luogo del re Roberto, che approfittò del fatto che, dal punto di vista del papa, l'impero era vacante.

\section{Vicario imperiale per conto del papa}

Il 16 luglio 1317, vacante imperio, papa Giovanni XXII nominò vicario imperiale d'Italia - Genova esclusa - Roberto d'Angiò. ${ }^{26}$ La cancelleria papale comunicò

21 I pisani assicurarono a re Roberto di fornire del supporto navale: «ebbe il re da' Pisani che quando facesse generale armata, gli darebbono $\mathrm{V}$ galee armate, o la moneta che costassono» (Villani, Nuova cronica, X 82, p. 287).

22 Caggese, Roberto d'Angiò, vol. II, p. 21.

23 Riezler, Vatikanische Akten, p. 49, n. 65 (18 giugno 1317): «tu itaque attento, quam dispendiosam sit etiam quibuscumque potentibus, sed tibi precipue, hoc tempore impacato guerrarum inculcare discrimina».

24 Cognasso, L'unificazione, p. 105.

25 Davidsohn, Geschichte, III, p. 611.

26 MGH, Const. V, n. 443A, pp. 367-368. La ricerca sul vicariato imperiale fu dominata per tanto tempo dal libro di Ercole, Dal comune al principato. Recentemente Zorzi, Ripensando $i$ vicariati, ha presentato la ricerca sul vicariato sottoponendo il fenomeno a una rivalutazione. Alla sua bibliografia si aggiunge, però, Baethgen, Der Anspruch des Papsttums, pp. 241-ss. Per quanto riguarda il vicariato imperiale durante il secolo XIV, cfr. in generale Heckmann, Stellvertreter, pp. 422-426; Viora, A proposito del vicariato, tratta più precisamente del vicariato di Roberto. 
la decisione anche a Firenze in una lettera in cui si sottolineavano i vantaggi che ne sarebbero risultati anche per la città toscana: «quanta ex regis prefati regimine accresceret utilitas quantusque in diversis mundi partibus favor accedet». Ma, allo stesso tempo, il papa ammoniva i suoi alleati: «in omnibus, que ad ipsius spectabunt officium parea[nt] humiliter et plenarie intenda[nt], ut exinde reipublice tranquillitas optate serenitas arrideat et tocius contratte securitas sperata resurgat». ${ }^{27}$

In questo modo Firenze si trovò ad essere sottomessa a Roberto per due ragioni: da un lato Roberto rimaneva il signore a termine, ${ }^{28}$ dall'altro, dal momento che la città toscana faceva comunque parte del regno d'Italia, il re di Napoli, una volta nominato vicario imperiale ne diveniva anche il sovrano per così dire "costituzionale".

Già poco tempo dopo, tuttavia, si aprì un primo contrasto tra il sovrano pontefice e il suo vicario. Quest'ultimo, come chiarito dal classico studio di Romolo Caggese, percepiva la carica di vicario imperiale come una carica redditizia: Roberto si dichiarò incapace di esercitare il suo ufficio in mancanza dei mezzi finanziari adeguati. ${ }^{29}$ Papa Giovanni, invece, riteneva l'appannaggio percepito da Roberto assolutamente sufficiente; ${ }^{30}$ secondo Caggese, Giovanni trascurava il fatto che il vicario perseguiva comunque gli interessi della Chiesa in Toscana e altrove. Ad ogni modo il papa non esaudì le richieste del re che lui stesso, prima di diventare papa (quando tanti signori richiedevano quella carica), ${ }^{31}$ aveva chiesto di nominare vicario imperiale. ${ }^{32}$

Come soluzione Giovanni propose di rivolgersi ai fiorentini e agli altri Guelfi:

27 MGH, Const. V, n. 443B, p. 368, rr. 27-29, 35 ss.

28 Monti, Sul dominio di Roberto, p. 162. Questa signoria non è stata oggetto di uno studio approfondito e soprattutto recente. De Vincentiis, Le signorie angioine, discute la ricerca più recente. Secondo Davidsohn, Geschichte, III, p. 531, questa signoria avrebbe potuto diventare “eterna”. A Genova, questa permanenza della signoria avvenne esattamente tramite la presenza militare degli angioini (cfr. Abulafia, Genova angioina, p. 17).

29 Caggese, Roberto d'Angiò, vol. II, pp. 22-25. Cfr. anche Tabacco, La casa di Francia, pp. 162-164, che ritiene la carica onerosa.

3018 dicembre 1317 (Preger, Die Anfänge, n. 36, p. 199-201, a p. 201).

31 Preger, Die Anfänge, n. 36, pp. 199-201, a p. 201: «nedum non petentes subsidium aliquod, quinimo grande nobis in promptu pro illo dare servicium offerentes, quin etiam a fide dignis audivimus, quod pridem pro vicariatu unici comitatus in Lombardia obtinendo data fuerunt florenorum decem milia impetranti».

32 Preger, Die Anfänge, n. 36, pp. 199-201, a p. 201: «scimus etiam quod tu ad predictum vicariatum obtinendum iam dudum ferventer aspirans a dicto predecessore nostro tibi illum concedente nullum petiisti subsidium, immo de sola concessione te valde reputasti et reputare debuisti contentum». 
in Florentina civitate vel alibi (...) Guelforum ambassiatores et nuncios, quasi cum eis de alio negocio tractaturus ad tuam presenciam evocares, et forte tua sibi circa vicariatum huiusmodi viriliter assumendum voluntate secreto detecta, eos ad subveniendum tibi exuberanter de pecunia et gente promptos et voluntarios invenires. ${ }^{33}$

Quando Filippo di Valois, nel 1321, scese in Italia come vicario di re Roberto per combattere insieme alle truppe del legato papale Bertrand du Pouget contro i “tiranni” di Milano, le città di Firenze, Bologna e Siena promisero di fornire 1000 cavalieri. Firenze, però, fu poco incline a legarsi per troppo tempo alla campagna del Valois. ${ }^{34}$

Giovanni non dimenticò questa riserva fiorentina e ricordò ai fiorentini la loro responsabilità nella ritirata del Valois: «quam graviter dudum in adventu dilecti filii nobilis viri Philippi de Valesio tarda vestrorum equitum missio et revocatio celeris obfuit». 35

\section{Genova e l'alleanza guelfa}

Il primo campo d'osservazione, nel nostro contesto, è la Liguria, e Genova in primo luogo. Nel 1318 gli Spinola e i Doria, entrambi cacciati da Genova, si rivolsero ai Visconti. ${ }^{36}$ Gli intrinseci di Genova cercarono invece appoggio presso il re di Sicilia, offrendogli di diventare signore di Genova. Una volta accettata questa carica, Roberto mandò in Liguria 300 cavalieri e vi giunse in persona il 23 luglio. A Genova fu investito della signoria per dieci anni - assieme al papa, che pure avrebbe preferito non essere coinvolto in maniera così esplicita. ${ }^{37}$

33 Preger, Die Anfänge, n. 36, pp. 199-201, a p. 201. Davidsohn, Geschichte, III, p. 624, commenta che i fiorentini furono ben capaci di rispondere a tali iniziative.

34 Cognasso, L’unificazione, p. 133; Davidsohn, Geschichte, III, p. 632; Tabacco, La casa di Francia, pp. 193-202.

35 Ficker, Urkunden, n. 22, pp. 13-14. L'abbandono da parte di Castruccio Castracani della Toscana, secondo Davidsohn, Geschichte, III, p. 641, rese possibile l'invio delle truppe fiorentine. Caggese, Roberto d'Angiò, vol. II, p. 52, al contrario, sostiene un'altra opinione.

36 Cognasso, L’unificazione, p. 118; Villani, Nuova cronica, X 87, pp. 294-295. Mollat, Les papes d'Avignon, p. 164, sostiene che la signoria di Genova fu affidata a Roberto per venti anni. In realtà, però, la signoria gli fu affidata per dieci anni e con un rinnovo di altri dieci anni dopo il ritorno di Roberto da Avignone (cfr. Villani, Nuova cronica, X 93, pp. 298-299; Caggese, Roberto d'Angiò, vol. II, p. 71). Per la storia di Genova nel secolo XIV e le sue relazioni con Avignone si veda Leonhard, Genua und die päpstliche Kurie, cap. 4.

37 Villani, Nuova cronica, X 93, pp. 298-299; Storie pistoresi, p. 103; Cognasso, L’unificazione, p. 120. Si presunse che Roberto accettò la signoria di Genova «a intenzione quando avesse a queto la signoria di Genova, si credea racquistare l'isola di Cicilia, e venire al di sopra di tutti gli suoi nimici» (Villani, Nuova cronica, X 94, p. 299). La ricerca di un collegamento senza 
Nonostante la sua iniziale riluttanza, papa Giovanni decise infine di aiutare i suoi alleati almeno in modo non militare: poiché si rifiutavano di abbandonare Genova, i Ghibellini furono scomunicati. ${ }^{38}$ Roberto convinse poi il papa a mettere a sua disposizione alcune navi papali che erano state preparate per una possibile crociata. L'appello all'ideologia guelfa, però, non fu sufficiente: Roberto dovette anche sottolineare che le navi non sarebbero state utilizzate altrimenti durante l'inverno. ${ }^{39}$ Finì che il papa non poté servirsi delle sue navi, perché furono affondate durante una battaglia navale assieme a quattordici navi angioine. ${ }^{40}$

Nel frattempo l'alleanza guelfa, con Firenze tra gli altri, mise a disposizione le sue truppe dal 1318 al $1320 .{ }^{41}$ Le richieste del capo militare dell'alleanza guelfa arrivarono a Firenze immediatamente dopo il prolungamento della signoria robertina. ${ }^{42}$ Roberto chiese a Firenze perfino di rompere la pace conclusa da lui stesso per facilitare la sua iniziativa genovese: Castruccio Castracani si stava sempre più dimostrando un nemico minaccioso. ${ }^{43}$ Del resto anche lui, come Roberto, era vicario imperiale, investito nel 1320 da Federico d'Asburgo. ${ }^{44} \mathrm{Da}$

interruzioni tra le terre angioine di Provenza e d'Italia, nonché di un collegamento sicuro per mare che avrebbe garantito questa continuità territoriale, fu sicuramente una delle ragioni che spinsero Roberto ad accettare la signoria (cfr. Abulafia, Genova angioina, p. 18). Heckmann, Stellvertreter, p. 424, suppone inoltre che "l'acquisto" di Genova fu una reazione al conferimento del vicariato imperiale da parte del papa, che tralasciò proprio Genova.

38 Davidsohn, Geschichte, III, p. 626. Bock, Studien zum politischen Inquisitionsprozess, non menziona questa scomunica. Parent, Dans les abysses, in conformità alla disponibilità delle fonti, si occupa piuttosto del periodo dopo il 1320, però accenna a questo atto alle pp. 66-ss. Si veda egualmente Parent, Publication et publicité.

39 Secondo Housley, The Italian Crusades, p. 100, nota 18, Roberto insisté sul fatto che «quod ipsarum armatio galearum propter instantiam proximam temporis yemalis esset inutilis et inanis in partibus tam remotis».

40 Housley, The Italian Crusades, pp. 100-101. Cfr. anche Abulafia, Genova angioina, p. 20.

41 Marchionne di Coppo Stefani, Cronaca fiorentina, n. 334, p. 125; Cognasso, L'unificazione, p. 121. Per la provenienza dei mercenari cfr. Davidsohn, Geschichte, III, p. 621. Una vittoria riportata dai mercenari messi a disposizione del re di Sicilia e Napoli fu celebrata a Firenze come una vittoria propria. Roberto, da parte sua, giustificò la sua domanda di aiuto militare tramite il pericolo siciliano: dichiarò di temere «Siculi hostis impii sagacitatem» e di non poter essere sicuro «de quibusdam Neapolitanis civibus, quod inficit imperialis macula» (MGH, Const. V, n. 505, pp. 408-09, rr. 34-35).

42 Caggese, Roberto d'Angiò, vol. II, p. 58; Cognasso, L'unificazione, pp. 125-126. I fiorentini, invece, si impegnarono militarmente nelle lotte di Brescia per conto proprio, senza il coinvolgimento del papa o del re (cfr. Davidsohn, Geschichte, III, pp. 627-628).

43 Davidsohn, Geschichte, III, pp. 624-625; 642.

44 MGH, Const. V, n. 570, p. 458, n. 573, p. 460. Lodovico IV l'istituì di nuovo il 29 marzo 1324 (cfr. n. 925, p. 764). 
Genova Roberto si recò allora ad Avignone per continuare la lotta a fianco del papa - che aveva infatti finito per farsi coinvolgere: già il 26 giugno 1319 il re scrisse ai Bresciani che papa Giovanni aveva assunto la guida della guerra, e che era deciso a non cedere ai nemici. ${ }^{45}$

$\mathrm{Fu}$ in questo quadro che Matteo Visconti e i suoi figli furono scomunicati per la prima volta il 4 gennaio 1318; altre scomuniche contro di loro e contro altri signori ghibellini sarebbero seguite a breve. ${ }^{46}$ Contro questa spregiudicata strumentalizzazione dell'azione spirituale si sarebbe scagliato Dante nel Paradiso: nel cielo di Giove al culmine della visione dell'aquila, simbolo della giustizia divina in terra, il poeta invoca l'intervento divino affinché «rimiri / ond'esce il fummo che 'l tuo raggio vizia», e soprattutto affinché si adiri «del comperare e vender dentro al templo / che si murò di segni e di martìri» (vv. 119-120; 122-3). Il riferimento a Giovanni XXII si fa quasi esplicito nelle aspre terzine successive, con tutta probabilità memori della scomunica comminata dallo stesso papa a Cangrande nel 1317: «già si solea con le spade far guerra; / ma or si fa togliendo or qui or quivi / lo pan che 'l pio Padre a nessun serra. / Ma tu che sol per cancellare scrivi, / pensa che Pietro e Paulo, che moriro / per la vigna che guasti, ancor son vivi» (vv. 127-132). Il canto si chiude con una sermocinatio di violentissimo cinismo, che vede il papa disconoscere Pietro e Paolo in favore di Giovanni Battista - la cui effigie era impressa sui fiorini (vv. 133-136).

\section{Lotte in Umbria e in Lombardia}

Anche in Umbria la situazione non andò del tutto come sperato, dal momento che i Ghibellini conquistarono Spoleto nel novembre del 1319. Perugia, alleata tradizionale di Firenze, si trovò così isolata in una sorta di accerchiamento ghibellino e bisognosa di aiuto. Giovanni tentò allora di spingere Firenze in suo soccorso tramite alcune lettere, ma soprattutto tramite la nomina di Bartolomeo de' Bardi, appartenente all'eminente società bancaria dei Bardi, a vescovo di Spoleto. L'investitura di Bartolomeo non poteva prescindere dalla riconquista

45 Caggese, Roberto d'Angiò, vol. II, pp. 49-50, ma cfr. anche p. 73: «il re (...) aveva pensato che la campagna contro i Visconti, contro i Ghibellini di Genova, contro i Savoja, contro Can Grande, contro Castruccio poteva esser condotta efficacemente soltanto da Avignone, e gli era sembrato indispensabile mobilitare gl'interessi della Chiesa e quelli del vecchio Pontefice». Il viaggio per Avignone era stato già progettato diverso tempo prima ma fu rinviato più volte (cfr. Tabacco, La casa di Francia, pp. 176-78).

46 Cognasso, L’unificazione, p. 116; Bock, Studien zum politischen Inquisitionsprozess, pp. 2156; e soprattutto Parent, Dans les abysses; Parent, Publication et publicité. 
di Spoleto - e, nei piani del papa, tale riconquista doveva essere condotta dai fiorentini.

Nel 1321 grazie alla loro partecipazione alla guerra contro Federico da Montefeltro e contro le città di Spoleto, Urbino, Osimo e Recanati, i fiorentini ottennero dal papa l'indulgenza di una crociata che sarebbe durata fino al $1323 .{ }^{47}$ Durante questa crociata i mercanti fiorentini realizzarono addirittura dei guadagni grazie alle bandiere dell'esercito crociato. Fu una delle prime crociate del secolo XIV contro i Ghibellini: la bolla relativa fu emessa il 8 dicembre 1321. Il mezzo politico delle crociate, tuttavia, non fu utilizzato senza limiti: l'interesse papale era decisivo, e Giovanni non cedeva sempre alle richieste angioine di predicare una crociata contro i nemici dei Guelfi; dal canto loro gli Angioini non si impegnarono in ogni crociata in Italia. Ciò non toglie che «the list of crusades which were waged by the papacy and its royal vassals together remains striking». ${ }^{48}$

Anche in Lombardia le lotte si protrassero: nel 1321 Firenze e Bologna contribuirono in maniera straordinaria a rinforzare le truppe guelfe - più che il papa e il re avrebbero dovuto contribuire servendosi di risorse più grandi. ${ }^{49}$

Quanto al contributo specifico del papa, i processi contro i Ghibellini - i Visconti e altri- proseguirono. ${ }^{50}$ Durante gli interrogatori un accusato sostenne di essere stato reclutato per praticare un maleficio nei confronti del papa, e spiegò che il suo mandante aveva evocato Dante come possibile rimpiazzo nel caso in cui lui avesse finito per non compiere il sortilegio. ${ }^{51} \mathrm{Da}$ maggio a novembre 1322 le tensioni si intensificarono tanto che Galeazzo Visconti fu costretto ad abbandonare Milano. Sulle sponde dell'Arno e del Rodano si sperava che una così dispendiosa guerra stesse ormai per concludersi, ${ }^{52}$ ma la gioia per questo successo durò poco: l'11 dicembre Galeazzo rientrò, e il 29 dicembre fu proclamato signore.

Le battaglie dell'anno 1323 videro alterni vincitori: in giugno i Guelfi entrarono nei borghi milanesi. Nel campo milanese i mercenari tedeschi erano però scontenti del mancato pagamento del soldo e minacciarono di consegnare Milano e Galeazzo Visconti al legato Bertrand du Pouget. Bertoldo di Neuffen arrivò in aiuto con 600 mercenari, assunse il governo della città e re-investì il Visconti

47 Davidsohn, Geschichte, III, pp. 665-669. Giovanni XXII fece predicare questa crociata persino in Renania.

48 Housley, The Italian Crusades, pp. 25-32, cit. a p. 32.

49 Davidsohn, Geschichte, III, pp. 656-666.

50 Cognasso, L'unificazione, pp. 157-158.

51 Parent, Dans les abysses, pp. 70-80.

52 Cognasso, L'unificazione, pp. 166-169. 
del ruolo di protector et defensor. ${ }^{53}$ Anche Bertoldo e, di conseguenza Ludovico IV, divennero così bersaglio degli inquisitori, e furono accusati di sostenere l'eresia perché avevano aiutato i Visconti scomunicati. ${ }^{54}$ Nonostante il grande margine d'azione di cui godevano gli inquisitori, i Guelfi dovevano ormai battere in ritirata. ${ }^{55}$

Dopo la grave sconfitta di Vaprio d'Adda, ${ }^{56}$ Giovanni rese pubblici i processi contro gli eretici milanesi ${ }^{57}$ e chiese ai fedeli della Chiesa, Firenze in primis, di partecipare a una crociata contro i nemici della fede ${ }^{58}$ e di fare donazioni in tutte le chiese per contribuire al suo finanziamento. Firenze, disperata per lo sviluppo della guerra e minacciata nel suo territorio, voleva ritirarsi dal conflitto. ${ }^{59}$ Fino alla discesa di Lodovico IV questi sviluppi segnarono un arresto dell'intera iniziativa militare guelfo-angioina in Lombardia. ${ }^{60}$

Nel cuore dell'universo guelfo, la Curia Romana, papa Giovanni cambiò infine opinione sul suo «maggior protettore» - come lo definì Cognasso: ${ }^{61}$ da questo momento in poi le sue speranze furono rivolte piuttosto a Parigi che a Napoli. Sul suo ospite, che sarebbe ripartito da Avignone poco dopo, disse:

53 Villani, Nuova cronica, II, X 212, pp. 394-396; Davidsohn, Geschichte, III, p. 687; Cognasso, L'unificazione, pp. 174-176. Il giuramento di fedeltà di Milano nei confronti del vicario imperiale di Lodovico IV, Bertoldo di Neuffen, si trova in MGH, Const. V, n. 752, pp. 586-588.

54 MGH, Const. V, n. 897, pp. 711-712 (12 aprile 1324). Cfr. Parent, Publication et publicité; Thomas, Ludwig der Bayer, pp. 118-121; Decker-Hauff, Graf Berthold von Neuffen, pp. 33-35; Serck, Berthold von Neuffen, pp. 14-22. Questo personaggio sarà finalmente più conosciuto grazie alla tesi di dottorato di Mirjam Eisenzimmer (Freie Universität Berlin / München): Berthold von Neuffen, Graf von Graisbach und Marstetten (1282-1342). Ein Berater Kaiser Ludwigs des Bayern. 55 Quando un numero elevato di cavalieri tedeschi cambiò fronte e si mise a combattere per la Chiesa, Castruccio Castracani e altri signori ghibellini lombardi portarono la guerra sotto le mura di Milano perché temevano che il loro potere fosse minacciato da un'eventuale vittoria guelfa (cfr. Davidsohn, Geschichte, III, p. 688).

56 Cognasso, L'unificazione, pp. 176-178.

57 Riezler, Vatikanische Akten, n. 356, p. 175. Gli atti dei processi furono inviati perfino in Inghilterra per evitare che Galeazzo Visconti ricevesse appoggi internazionali (cfr. Riezler, Vatikanische Akten, n. 360-361, pp. 176-177, e anche, come sempre, Parent).

58 Il 24 agosto 1324 Giovanni XXII chiese a Firenze, già ampiamente coinvolta nella guerra, di fornire altre truppe (cfr. Ficker, Urkunden, n. 25, p. 15 [= MGH, Const. V, n. 780, pp. 607608]; cfr. anche Riezler, Vatikanische Akten, n. 330, pp. 166-167).

59 Davidsohn, Geschichte, III, p. 317: Firenze non voleva «für den lombardischen Krieg (...) immer weitere Aufwendungen an Geld und Menschen machen». A questo contesto appartiene probabilmente anche il documento pubblicato in Ficker, Urkunden, n. 31, p. 17 (23 giugno 1324).

60 Léonard, Les Angevins de Naples, p. 238.

61 Cognasso, L’unificazione, p. 183. 
certe, nos fuimus et sumus decepti in isto misero rege Roberto, qui est miser et miserabilis. Sperabamus enim et indubitanter credebamus, quod dictum negocium una cum Januensibus assumeret, precipue cum ipse in hoc videat finalem suam et suorum destructionem. Set ipse est ita timorosus et miserabilis, quod non est ausus hoc acceptare. Et ideo habeat sibi! ${ }^{62}$

\section{Ultime lotte in Toscana fino ad Altopascio}

In Toscana, nel frattempo, Castruccio Castracani continuava a costituire una minaccia, e anche su questo campo la guerra rimase a lungo aperta. Giovanni XXII era ampiamente coinvolto: a seguito di alcune manovre poco onorevoli criticò i fiorentini, accusandoli di essere molli e di dare un cattivo esempio agli alleati. ${ }^{63}$ Dopo la cacciata del vicario angioino da Pistoia, però, si rivolse di nuovo a Firenze per chiedere aiuto. ${ }^{64}$

Queste sconfitte della parte guelfa in Lombardia e in Toscana incoraggiarono ulteriormente Castruccio Castracani. ${ }^{65}$ La minaccia di un nemico così vicino rese pressante la necessità di trovare un generale capace: di Bertrand de Beaux, ${ }^{66}$ ritenuto incapace, si tentò di liberarsi già dal $1323 .{ }^{67}$ Ramon de Cardo-

62 Acta Arag. II, n. 392, pp. 611-614, citazione alle pp. 612-613. Il rapporto che menziona questo moto di sdegno di Giovanni è datato 23 gennaio 1324, dunque prima della partenza di Roberto per Napoli. Tuttavia Léonard, Les Angevins de Naples, p. 243, non vuole attribuire a questo documento una grande importanza: «n'exagérons pas l'importance d'un mouvement d'humeur, soigneusement rapporté, sinon provoqué, par le représentant d'un pays mal disposé pour la cour de Naples, comme fidèle à la cause gibeline et contraint à une participation onéreuse et peu honorable aux expéditions napolitaines contre la Sicile: il n'y avait pas moins là l'indice d'une divergence certaine entre les Angevins et Avignon». Il malcontento, però, non fu sempre dalla parte del papa: anche Roberto fu talvolta esasperato dall'alleato papale, come dimostra Tabacco, La casa di Francia, p. 175. Per una valutazione del soggiorno avignonese del re Roberto si confrontino le opinioni di Caggese, Roberto d'Angiò, vol. II, pp. 73-74; Tabacco, La casa di Francia, p. 281; Léonard, Les Angevins de Naples, p. 243; Barbero, Il mito angioino, pp. 151-152; Galasso, Il Regno di Napoli, p. 139. La domanda fondamentale resta quella relativa al successo del tentativo di Roberto di guidare la lotta insieme al papa.

63 Cfr. Preger, Die Anfänge, n. 156, p. 264 (18 agosto 1323): i fiorentini avrebbero avuto l'occasione di annientare il nemico, ma per inerzia non riuscirono a coglierla.

64 Caggese, Roberto d'Angiò, vol. II, p. 62 con nota 3. Pistoia fu vinta da Castruccio Castracani (ivi, pp. 62-63).

65 Cognasso, L'unificazione, p. 187; Caggese, Roberto d'Angiò, vol. II, p. 75.

66 Villani, Nuova cronica, X 254, p. 427; Davidsohn, Geschichte, III, p. 587. Quanto al personaggio, si veda la voce di J. Göbbels per il DBI (<http://www.treccani.it/enciclopedia/bertrandodel-balzo_res-12cf9dfa-87ec-11dc-8e9d-0016357eee51_(Dizionario-Biografico)>.

67 Caggese, Roberto d'Angiò, vol. II, p. 76, nota 1. Si rinunciò ad attaccare Lucca perché i fiorentini si convinsero che Bertrand, cognato del re, fosse incapace di comandare con succes- 
na, comandante guelfo nella sconfitta di Vaprio d'Adda, giunse il giorno dopo la conquista di Pistoia a opera di Castruccio Castracani ${ }^{68}$ e assunse la carica di nuovo capitano generale della guerra. ${ }^{69}$

Nonostante questo inizio poco incoraggiante, il fronte ottenne alcuni successi, tanto che addirittura si diffuse la speranza di arrivare alla vittoria decisiva. ${ }^{70}$ Le belle speranze della signoria fiorentina sembravano sul punto di rivelarsi ben riposte, quando giunse invece la sconfitta di Altopascio. ${ }^{71}$ Insieme alla sconfitta dei bolognesi a Zappolino queste «due battaglie segnarono il crollo della egemonia guelfa in Italia» - dice Cognasso. ${ }^{72}$ Giovanni XXII inviò una lettera consolatoria e piena di auspici di vittorie future ${ }^{73}$ - che sarebbero invece mancate a lungo. Da Napoli non c'era da aspettarsi molto, perché Roberto era immerso nei preparativi per il futuro tentativo di riconquistare il regno di Trinacria. ${ }^{74}$ Ludovico IV, dopo la riconciliazione di Trausnitz, poteva finalmente aspirare alla corona imperiale - il che l'avrebbe portato, secondo le previsioni dei

so un esercito composto di contingenti provenienti da Bologna, Siena e da altri signori alleati e segnato da forti divergenze d'opinione. Queste divergenze seguivano le opposizioni "sociali" tra magnati e popolani (cfr. Davidsohn, Geschichte, III, p. 696). Un'altra ragione di controversia tra Bertrand e Firenze fu la valutazione strategica della conquista del castello Carmignano (cfr. ivi, p. 713; Marchionne di Coppo Stefani, Cronaca fiorentina, n. 372, p. 136).

68 Sulla conquista di Pistoia cfr. Villani, Nuova cronica, X 294, pp. 459-460; sull'arrivo di Ramon de Cardona cfr. ivi, X 295, p. 460. Conquistando Pistoia, Castruccio realizzò il suo vicariato imperiale (cfr. MGH, Const. V, n. 927, p. 766).

69 Dopo la sconfitta di Vaprio d'Adda Ramon aveva dovuto giurare di non combattere mai più in territorio italiano (cfr. Cognasso, L'unificazione, p. 179); ma il giuramento ebbe come testimone e garante uno scomunicato, e fu perciò ritenuto nullo. Ramon si fece poi confermare ulteriormente da re Roberto (cfr. Caggese, Roberto d'Angiò, vol. II, p. 76; Villani, Nuova cronica, X 295, p. 460). Giovanni XXII scrisse allora ai fiorentini per incitarli a pagare un soldo adeguato e a fornire delle altre truppe, e raccontò che Ramon non aveva accettato la carica a cuor leggero (cfr. Preger, Reinkens, Die Verträge, n. 202, pp. 161-62 [21 gennaio 1325]; cfr. anche Ferrer Mallol, Cavalieri catalani).

70 Decisiva soprattutto la conquista del castello di Artimino il 22 maggio 1325 (cfr. Villani, Nuova cronica, X 300, p. 463-464); sulla leva di mercenari, cfr. ivi, X 302, dove si trova un amaro commento relativo alle truppe fornite dagli alleati: «essendo i Fiorentini in vittoria, tutti gli amici mandarono soccorso».

71 Davidsohn, Geschichte, III, pp. 735-738; Partner, Lands of St Peter, p. 317, ritiene che per qualche tempo fu Bologna la cerniera dell'alleanza guelfa.

72 Cognasso, L'unificazione, p. 163.

73 Davidsohn, Geschichte, III, p. 743. Forse la lettera riportata in Ficker, Urkunden, n. 39, p. 23 (13 novembre 1325) appartiene a questo contesto.

74 Davidsohn, Geschichte, III, p. 745. Ė vero che Roberto fu occupato dai preparativi per la guerra e non inviò le sue truppe a Firenze, ma Carlo di Calabria, suo figlio, inviò una missiva alle città alleate promettendo un aiuto veloce (cfr. Caggese, Roberto d'Angiò, vol. II, p. 80). 
fiorentini, anche a Firenze. Nel frattempo il Castracani fu libero di devastare il contado fiorentino e arrivò fino a Fiesole. ${ }^{75}$

\section{Un primo riepilogo}

Le relazioni angiono-papali-fiorentine non furono mai interamente prive di tensioni né di interessi divergenti, ma con queste sconfitte e con il mancare del sostegno napoletano la situazione cominciò a evolvere in direzione di un ribaltamento delle alleanze: Giovanni XXII si sarebbe infine alleato col re di Boemia, ${ }^{76}$ i fiorentini e Roberto avrebbero combattuto l'alleato papale ${ }^{77} \mathrm{e}$, alcuni decenni più tardi, Firenze avrebbe combattuto il papa stesso ${ }^{78}$ dopo che questi si fu ritirato per sempre dalle signorie angioine - come ha dimostrato Amedeo De Vincentiis. ${ }^{79}$

Il resoconto dei fatti condotto fin qui manca però di una componente fondamentale: l'azione robertina, e soprattutto il venir meno del suo contributo alle lotte dei fiorentini, non si comprende a fondo se non si considera il ruolo cruciale che la Sicilia rivestiva per il sovrano. La logica principale, se non l'unica logica, di tanta parte della sua politica ruotava infatti proprio attorno alla Sicilia: in nome di essa chiese un contributo ai pisani e poté incitare Genova a fornire delle navi per la riconquista dell'isola. In fondo i fiorentini, combattendo su richiesta di Roberto Castruccio Castracani in Toscana, contribuirono all'energico tentativo del re di Napoli di contrastare i Vespri Siciliani - operazione non per tutti legittima, se teniamo a mente che Dante, ad esempio, ne parla come della naturale conseguenza di una «mala segnoria» (Par. XVIII, 73-75). Una volta dissolte le minacce esterne con la morte di Enrico VII e l'allontanamento dei Ghibellini lombardi dal suo regno, l'impegno di Roberto nella lotta contro i nemici della parte guelfa in Toscana e in Umbria si raffreddò considerevolmente.

75 Villani, Nuova cronica, X 317-319, pp. 485-89; Caggese, Roberto d'Angiò, vol. II, pp. 80-81. Per la riconciliazione di Trausnitz cfr. Thomas, Ludwig der Bayer, p. 172-74; MGH, Const. VI, 1, n. 29, pp. 18-20.

76 Margue, La conquête de l'Italie du Nord; Härtel, Die Italienpolitik, pp. 363-382; Dumontel, L'impresa italiana; Landogna, Giovanni di Boemia.

77 Villani, Nuova cronica, XI 210, p. 775. Cfr. anche Caggese, Roberto d'Angiò, vol. II, pp. 149-ss. 78 Favier, Les papes d'Avignon, pp. 482-488.

79 De Vincentiis, Signorie angioine. Non ho potuto consultare la sua tesi di dottorato. 


\section{Modalità d'influenza e di sostegno}

Giovanni XXII, dal momento che al di fuori dello Stato della Chiesa era solo raramente signore di una città, non disponeva delle stesse capacità d'influenza di re Roberto. Il papa poteva inviare delle truppe, ma su questo terreno l'interlocutore principale di Roberto era il cardinale legato Bertrand du Pouget, che si trovava in Italia e aveva delle truppe già assoldate: ${ }^{80}$ al papa il re si rivolgeva soltanto se il legato non corrispondeva alle richieste fiorentine ${ }^{81}$ La repubblica fiorentina, da parte sua, si rivolse a più riprese al papa per chiedergli di predicare una crociata contro i nemici di Firenze - una richiesta che non fu esaudita se non raramente. ${ }^{82} \mathrm{Il}$ tentativo di indurre il papa ad aprire dei processi di eresia contro i nemici della repubblica giunse vicino al suo obiettivo: anche in questo caso si trattò di una coincidenza di interessi che si poteva realizzare solo poche volte, e non sempre o non necessariamente nei momenti in cui l'azione era richiesta. ${ }^{83} \mathrm{Gli}$ atti dei processi furono pubblicati su scala europea - ed è anche per giustificare gli appelli alla crociata che Giovanni arrivò a compiere questo passo. ${ }^{84}$

La repubblica fiorentina chiedeva anche la tassazione del clero fiorentino: poiché le truppe fiorentine difendevano non soltanto l'area urbana e il contado ma anche il clero, questo avrebbe dovuto, secondo la signoria fiorentina, contri-

80 Per questo personaggio si veda la voce corrispondente di A. Jamme, P. Jugie nel DBI (<http://www.treccani.it/enciclopedia/bertrando-del-poggetto_(Dizionario-Biografico) >.

81 Per esempio il 23 maggio 1328: «supplicamus, quatenus omni mora sublata scribere dignemini efficaciter domino Lombardie legato nec non communibus Bononie et aliarum civitatum fidelium Lombardie, quod ad nos dicta causa omne suum et ecclesie exfortium destinent indilate, et hoc facto faciant et non verbis» (Ficker, Urkunden, n. 119, p. 72).

82 Ficker, Urkunden, n. 57, p. 35 (= MGH, Const. VI, 1, n. 284, pp. 200-201): «primo nanque petiit ambaxiator predictus, quod cum Ludovicus olim Bavarie, tractatibus et colloquiis iniquis cum hereticis et rebellibus habitis ac pactionibus confederationibus et colligationibus initis dampnabiliter cum eisdem ad offendendum deum et ecclesiam ac fideles, Lombardiam dampnate intentionis proposito sit ingressus, tam ad ipsum quam dictos rebelles et hereticos eorumque fautores et sequaces citius et felicius extirpandos crucem predicari contra eos faceremus. Super quibus duximus respondendum, quod cum fratribus nostris deliberatione super hiis habita pleniori annuente domino proponimus, quod expediens in hac parte videbitur ordinare». Cfr. anche Housley, The Italian Crusades, pp. 145-47. Giovanni, nonostante essendo il papa perseguisse più energicamente questa strada, tentennò a lungo, giacché preferiva combattere sul campo spirituale (cfr. Bock, Studien zum politischen Inquisitionsprozess, p. 31).

83 Parent, Publication et publicité, pp. 104; 121-128.

84 Parent, Publication et publicité, pp. 96-97; 102; 113-114. Housley, The Italian Crusades, p. 69, commenta così la pubblicazione: «the most important aim of the papacy in justifying the Italian crusades was to present the struggle in Italy as an issue which affected all Christians». 
buire «pro novorum constructione murorum». Una richiesta che, una volta esaudita, portò all'interdetto contro la città guelfa. ${ }^{85}$ La repubblica domandò anche un appoggio per una "guerra economica": il papa, nelle richieste di Firenze, avrebbe dovuto esortare i vescovi di Siena e Volterra a distruggere le strade verso Pisa e Lucca e a interrompere ogni commercio con questi comuni. Una simile richiesta avrebbe dovuto però piuttosto essere diretta al re di Francia. $^{86}$

Oltre a tutte queste misure che si potrebbero chiamare "pratiche", lo strumento principale con il quale il papa fornì il suo appoggio fu un'epistola contenente consigli paterni: in essa Giovanni XXII pregò i fiorentini di non farsi spaventare dalla discesa di Ludovico IV e di fare ulteriori sforzi; ${ }^{87}$ alcune settimane più tardi fornì addirittura un consiglio tattico: bisognava incrementare il numero di cavalieri e non spargere le truppe.

Providenciam et universitatem vestram rogamus, monemus et hortamur attente, vobis nichilominus paterno consilio suadentes, quatenus sic expedienti bellatorum exfortio vestrum roboretis exercitum, vires vestras undecunque dispersas reducentes in unum, cum unita virtus fiat fortior disgregata, quod, si opus extiterit, potenter eisdem inimicis vestris et emulis resistere valeatis, circa quod nostrum auctore deo non deerit auxilium et consilium oportunum. 88

\section{Conclusioni}

Concentrandoci sull'ultimo quinquennio della vita di Dante abbiamo seguito il coinvolgimento della repubblica fiorentina nella politica papale, ma soprattutto robertina, con le notevoli differenze d'interesse relative alle due. ${ }^{89}$ Questi anni,

85 Villani, Nuova cronica, XI 110, p. 662; cfr. Marchionne di Coppo Stefani, Cronaca fiorentina, n. 447, p. 159; Davidsohn, Geschichte, III, p. 865. La lettera papale non si trova nelle pubblicazioni consuete.

86 Ficker, Urkunden, n. 178, pp. 101-102 (8 settembre 1328); Mommsen, Italienische Analekten, n. 185, pp. 82-83 (7 marzo 1329), n. 188, pp. 83-84 (16 maggio 1329). Firenze stessa sembra non aver rispettato le proprie proposte (cfr. Pinto, Aspetti della guerra, p. 236).

87 Ficker, Urkunden, n. 61, pp. 37-38.

88 Riezler, Vatikanische Akten, n. 878, pp. 334-335.

89 Maire Vigueur, Nello Stato della Chiesa, p. 771: «a due riprese perlomeno, all'epoca di Clemente V e di Benedetto XII, ci furono tra Firenze e gli Angioini, da una parte, e il papato, dall'altra, contrasti con serie ripercussioni sulla scelta degli ufficiali, là dove i capofila del guelfismo dettavano legge o per lo meno esercitavano una forte influenza in questo campo»; a queste considerazioni possiamo dunque aggiungere altri contrasti durante la vita di Dante, che saranno destinati a inasprirsi nel futuro. Cfr. anche Tabacco, La casa di Francia, p. 335. 
fino alla discesa di Ludovico IV, sono l'ultimo periodo in cui l'alleanza guelfa classica, così come si era formata nel secolo XIII sotto Carlo d'Angiò, fu ancora attiva e vitale. Le azioni militari richieste dalle relazioni tra il papa e i fiorentini avrebbero dovuto portare ulteriori conseguenze, come scomuniche e guerre economiche, fino alla preparazione di un conflitto su scala - almeno teoricamente - europea. Il papa però non corrispose se non molto parzialmente alle esigenze fiorentine. $\mathrm{Ci}$ associamo, dunque, alla domanda implicitamente posta da Maire Vigueur: volendo analizzare il guelfismo come alleanza politica attiva, bisogna interrogarsi sulle condizioni precise di ogni comune: chi si associa quando, con chi, e per quali ragioni? ${ }^{90}$

90 Maire Vigueur, Nello Stato della Chiesa, pp. 772-774. 